\title{
A RATHER CLASSLESS MODEL
}

\author{
MATT KAUFMANN
}

ABSTRACT. Using $\diamond_{\omega_{1}}$, a model $\mathfrak{R}$ of Peano arithmetic is constructed which has a unique extension $(\Re, \chi)$ to a model of $\Delta_{1}^{1}-P A$, Peano arithmetic with $\Delta_{1}^{1}$ comprehension.

As in Barwise-Schlipf [2], " $\Delta_{1}^{1}$-PA" will refer to the theory PA (Peano arithmetic) with the axiom of induction for classes, and the axiom scheme of $\Delta_{1}^{1}$-comprehension which asserts the universal closure of

$$
\forall x(\Phi \leftrightarrow \Psi) \rightarrow \exists X \forall x(x \in X \leftrightarrow \Phi(x))
$$

for all "essentially $\Pi_{1}^{1}$-formulas" $\Phi(x)$ and all "essentially $\Sigma_{1}^{1}$ formulas" $\Psi(x)$. An essentially $\Pi_{1}^{1}$ formula is one in the smallest class containing all first-order formulas and closed under $\wedge, \vee, \forall x_{i}, \exists x_{i}$, and $\forall X_{i}$; similarly for an essentially $\Sigma_{1}^{1}$ formula. In the paper mentioned above, Barwise and Schlipf show that the nonstandard models $\mathfrak{N}$ of PA which can be expanded to models $(\mathfrak{N}, \chi)$ of $\Delta_{1}^{1}$-PA are exactly the recursively saturated ones; in particular, if $D F_{\mathfrak{R}}$ $=\left\{X \subseteq N:^{1} X\right.$ is definable ${ }^{2}$ with parameters in $\left.\mathfrak{R}\right\}$, then for recursively saturated models $\mathfrak{R}$ of PA, $\left(\mathfrak{R}, D F_{\mathfrak{R}}\right) \vDash \Delta_{1}^{1}$-PA. They also observe that if $\mathfrak{R}$ is a countable recursively saturated model of PA, then $\cup\left\{\chi:(\Re, \chi) \vDash \Delta_{1}^{1}-\mathrm{PA}\right\}$ has power $2^{k_{0}}$, using the version of Makkai's theorem in [1, IV.4]. Answering a question of Barwise, with the help of some much-appreciated conversations with him, we show that assuming $\diamond_{\omega_{1}}$, there is a (recursively saturated) model $\mathfrak{R}$ of PA such that $\left(\mathfrak{R}, D F_{\mathfrak{R}}\right)$ is the only expansion of $\mathfrak{R}$ to a model of $\Delta_{\mathrm{l}}^{1}$-PA. This follows as the corollary of the following

TheOREM $\left(\diamond_{\omega_{1}}\right)$. Suppose $\mathfrak{M}$ is a countable recursively saturated model of Peano arithmetic. Then $\mathfrak{M}$ has an $\omega_{1}$-like, recursively saturated, elementary end extension $\mathfrak{N}$ with the following property:

If $S \subseteq N$ such that for each $n \in N,\left\{x \in S: x<^{\mathfrak{N}} n\right\}$

is definable with parameters in $\Re$, then $S$ is

definable with parameters in $\mathfrak{N}$.

Received by the editors August 8, 1975.

AMS (MOS) subject classifications (1970). Primary 02H13; Secondary 02F27.

Key words and phrases. Recursively saturated model, Peano arithmetic, $\Delta_{1}^{1}$-comprehension, $\diamond_{\omega_{1}}$, Barwise Compactness Theorem, Barwise Completeness Theorem, $\mathrm{HYP}_{\mathfrak{R}}, \mathrm{KPU}^{+}$.

${ }^{1}$ Henceforth, $|\mathfrak{N}|=N,\left|\mathfrak{M}_{\alpha}\right|=M_{\alpha}$, and so forth.

2 "Definable" means "first-order definable". 
COROLlaRY $\left(\diamond_{\omega_{1}}\right)$. There is a recursively saturated model $\mathfrak{N}$ of $P A$ such that if $(\mathfrak{R}, \chi) \vDash \Delta_{1}^{1}-P A$, then $\chi=D F_{\mathfrak{R}}$.

Proof OF COROLLARY. Let $\mathfrak{M}$ be any countable recursively saturated model of PA, and let $\mathfrak{R}$ be as in the conclusion of the theorem. Certainly $D F_{\mathfrak{N}} \subseteq \chi$, since $\mathfrak{R} \vDash \mathrm{PA}$. To show $\chi \subseteq D F_{\mathfrak{R}}$, let $X \in \chi$. Then by using Gödel's $\beta$ function, for example, we see by induction that there is a code for $\left\{x \in X: x<^{\mathfrak{N}} n\right\}$ for each $n \in N$ (via some uniform coding). By the theorem, $X \in D F_{\mathfrak{R}}$, as desired.

Proof OF THEOREM. The proof uses the method of Keisler and Kunen [3] and the following results which can be found as indicated in Barwise [1]: Barwise Compactness Theorem (III5.6); Barwise (Extended) Completeness Theorem (III5.7); (Schlipf) $\mathfrak{N}$ is recursively saturated if and only if $H Y P_{\mathfrak{R}}$, the least admissible set with $\mathfrak{R}$ as a set of urelements, has ordinal $\omega$ (IV5.3); and for recursively saturated $\mathfrak{N}, D F_{\mathfrak{R}}=\left\{X \subseteq N: X \in H Y P_{\mathfrak{R}}\right\}$ (II7.2).

Let $\left\langle S_{\alpha}: \alpha<\omega_{1}\right\rangle$ be as in the definition of $\diamond_{\omega_{1}}$. We can assume that $\mathfrak{M}$ has universe $\omega$. We construct (as in Keisler [3]) an elementary end extension chain of recursively saturated models

$$
\mathfrak{M}=\mathfrak{M}_{0} \preccurlyeq_{e} \mathfrak{M}_{1} \preccurlyeq_{e} \cdots \preccurlyeq_{e} \mathfrak{M}_{\alpha} \preccurlyeq_{e} \cdots \quad\left(\alpha<\omega_{1}\right),
$$

where $M_{\alpha}=\alpha$ for limit ordinals $\alpha$, by induction on $\alpha$. Unless $\alpha=\gamma+1$ for some limit ordinal $\gamma$, we set $\mathfrak{M}_{\alpha}=\cup_{\beta<\alpha} \mathfrak{D}_{\beta}$, a process which preserves elementarity, end extensions, and recursive saturation. If $\alpha=\gamma+1$ for some limit $\gamma$, and $S_{\gamma}$ is definable in $\mathfrak{M}_{\gamma}$, let $L$ be a language containing constant symbols $\bar{n}$ for all $n \in \mathfrak{M}_{\gamma}$ and $\bar{m}$ for all $m \in \omega$, in an effective way, along with new constant symbols $c$ and $d$, binary function symbols + and $\cdot$, and a binary relation symbol $\in$. Consider the following theory $T$ formulated in the fragment of $L_{\infty \omega}$ given by $H Y P_{\mathfrak{R}_{\gamma}}$ :

$\mathrm{KPU}^{+}$(admissible set theory with a set of all urelements);

The first-order elementary diagram of $\mathfrak{M}$ (with urelement variables);

$\left\{\bar{n}<c: n \in M_{\gamma}\right\}$

$\wedge_{n \in M_{\gamma}} \forall p\left(p<\bar{n} \rightarrow \bigvee_{r \in M_{\gamma}}(p=\bar{r})\right)$;

" $d$ is a finite ordinal";

$\{\bar{m} \in d: m \in \omega\}$.

$T$ is obviously $\Sigma_{1}$ on $H Y P_{\mathfrak{R}_{\gamma}}$. Also, any $H Y P_{\mathfrak{R}_{\gamma}}$-finite subset of $T$ contains only finitely many sentences of the form " $d \in \bar{m}$ " for $m \in \omega$, and thus has a model since every model of PA has an elementary end extension and every model has a $H Y P$. By Barwise compactness, $T$ has a countable model; but its wellfounded part, which has ordinal $\omega$, is also admissible by the Truncation Lemma (see Barwise [1, II8.4]). Thus its urelement structure is recursively saturated: call it $\mathfrak{M}_{\alpha}$. Without loss of generality, $M_{\alpha}=\gamma+\omega$ and $\mathfrak{M}_{\gamma}$ $\prec_{e} \mathfrak{M}_{\alpha}$.

The remaining case occurs when $\alpha=\gamma+1, \gamma$ a limit, and $S_{\gamma}$ is not definable in $\mathfrak{M}_{\gamma}$. In that case we let $L$ and $T$ be as before but we want to take $\mathfrak{M}_{\alpha}$ to be a model of $T$ omitting the following set $\Sigma$ of formulas: 


$$
\left\{“ \bar{n} E x ": n \in S_{\gamma}\right\} \cup\left\{\neg \text { “ } \bar{n} E x ": n \notin S_{\gamma}\right\},
$$

where by " $\bar{n} E x$ " we mean " $\bar{n}$ is in the set coded by $x$." We choose our coding so that if $A \subseteq B \subseteq M_{\gamma}$ and $A$ and $B$ have codes in $\mathfrak{M}_{\gamma}$, then the code for $A$ is less than the code for $B$. It suffices to show that $T$ locally omits $\Sigma$. Suppose not; say

$$
\begin{aligned}
& T \vDash \forall x(\varphi(x) \rightarrow \text { “ } \bar{n} E x ") \text { for } n \in S_{\gamma}, \text { and } \\
& T \vDash \forall x(\varphi(x) \rightarrow \neg \text { “ } \bar{n} E x ") \text { for } n \notin S_{\gamma},
\end{aligned}
$$

where $\varphi(x)$ is in the fragment of $L_{\infty \omega}$ given by $H Y P_{\mathbb{R}_{\gamma}}$ and $\varphi(x)$ is consistent with $T$. Then

$$
n \in S_{\gamma} \Leftrightarrow T \vDash \forall x(\varphi(x) \rightarrow “ \bar{n} E x ")
$$

and

$$
n \notin S_{\gamma} \Leftrightarrow T \vDash \forall x(\varphi(x) \rightarrow \neg “ \bar{n} E x ") .
$$

Thus $S$ is both $\Sigma_{1}$ and $\Pi_{1}$ on $H Y P_{\mathfrak{R}_{\gamma}}$ by Barwise (extended) completeness. Since $S \subseteq M_{\gamma} \in H Y P_{\mathfrak{R}_{\gamma}}, S \in H Y P_{\mathfrak{R}_{\gamma}}$ by $\Delta_{1}^{-}$-separation. Therefore (by [1, II7.2]), $S_{\gamma} \in D F_{\mathfrak{P}_{\gamma}}$, a contradiction. So $\Sigma$ is locally omitted by $T$, and we can take $\mathfrak{M}_{\alpha}$ to be a model of $T$ as before $\left(M_{\alpha}=\gamma+\omega, \mathfrak{M}_{\gamma} \prec_{e} \mathfrak{M}_{\alpha}\right)$, except that here $\mathfrak{M}_{\alpha}$ also omits $\Sigma$.

Now that the chain is constructed, we check to see that $\mathfrak{R}=\cup_{\alpha<\omega_{1}} \mathfrak{M}_{\alpha}$ has the desired properties. $\mathfrak{R}$ is recursively saturated because it is the union of an elementary chain of recursively saturated models. Let $S \subseteq N$ such that for each $n \in N,\left\{x \in S: x<^{\mathfrak{N}} n\right\}$ is definable with parameters. $\left\{\gamma \in \omega_{1}:\left(\mathfrak{M}_{\gamma}, S\right.\right.$ $\left.\left.\cap \mathfrak{M}_{\gamma}\right) \prec(\Re, S)\right\}$ is easily seen to be closed and unbounded in $\omega_{1}$, as is $\left\{\gamma \in \omega_{1}: \gamma\right.$ is a limit $\}$. So if

$$
C=\left\{\gamma \in \omega_{1}: \gamma \text { is a limit and }\left(\mathfrak{M}_{\gamma}, S \cap \mathfrak{M}_{\gamma}\right) \prec(\mathfrak{R}, S)\right\},
$$

then $C$ is also c.u.b. Pick $\gamma \in C$ such that $S \cap \gamma=S_{\gamma}$, using $\diamond_{\omega_{1}}$. Then $\left(\mathfrak{M}_{\gamma}, S_{\gamma}\right) \prec(\mathfrak{N}, S)$. Pick any $n \in M_{\gamma+1}-M_{\gamma}$. Then $\left\{x \in S: x<{ }^{\omega_{1}} n\right\}$ is definable in $\mathfrak{N}$, so it has a code $r$ in $\mathfrak{N}$. By our choice of coding, $r<^{\mathfrak{N}}$ (the code for $\left.\left\{x \in N: x<^{\mathfrak{N}} n\right\}\right)$. But the latter is in $M_{\gamma+1}$, so since $\mathfrak{M}_{\gamma+1} \subset_{e} \mathfrak{N}, r$ $\in M_{\gamma+1}$. By construction of $\mathfrak{M}_{\gamma+1}, S_{\gamma}$ must be definable in $\mathfrak{M}_{\gamma}$. This same definition puts $S$ in $D F_{\mathfrak{R}}$, since $\left(\mathfrak{M}_{\gamma}, S_{\gamma}\right)<(\Re, S)$.

REMARK. The following "effective version" of part of Theorem B of Keisler [3] can be proved essentially as the above theorem is proved:

Assume $\diamond_{\omega_{1}}$. Let $\mathfrak{A}$ be a countable tree-like model which satisfies the collection scheme, and suppose $\mathfrak{A}$ is recursively saturated. Then $\mathfrak{A}$ has a recursively saturated end elementary extension $\mathfrak{B}$ such that $\mathfrak{B}$ is an $\omega_{1}$-like model and every branch of $\mathfrak{B}$ is definable in $\mathfrak{B}$.

Since (as Keisler shows) models of ZF and ZF minus infinity can be viewed as tree-like models, there is an obvious analogue of the theorem (proved above for PA) for models of these theories. 
[Added in proof October 19, 1976. S. Shelah has informed the author of an absoluteness result which implies that $\diamond_{\omega_{1}}$ can be eliminated from the hypothesis of the result here as well as in the Keisler-Kunen result. (See his AMS Notices of October 1975 and February 1976.)|

\section{REFERENCES}

1. K. J. Barwise, Admissible sets and structures, Springer-Verlag, Heidelberg, 1975.

2. K. J. Barwise and J. Schlipf, On recursively saturated models of arithmetic, Model Theory and Algebra, Lecture Notes in Math., vol. 498, Springer-Verlag, Berlin, 1976, 42-55.

3. H. J. Keisler, Models with tree structures, Proc. Tarski Sympos., Proc. Sympos. Pure Math., Amer. Math. Soc., Providence, R.I., 1974, pp. 331-348.

4. J. Schlipf, Some hyperelementary aspects of model theory, Doctoral Dissertation, University of Wisconsin, Madison, Wis., 1975.

Department of Mathimatics, University of Wisconsin, Madison, Wisconsin 53706 